Classification

Physics Abstracts

$03.40 \mathrm{G}-47.25 \mathrm{E}-02.70$

\title{
Computation of a thermal boundary layer including strong viscous-inviscid flow interaction
}

\author{
Pascale Kulisa $\left({ }^{1}\right)$, Francis Lebœuf $\left({ }^{1}\right)$, Philippe Klinger $\left({ }^{2}\right)$ and Jacques Bernard $\left({ }^{2}\right)$ \\ (') Ecole Centrale de Lyon, Laboratoire de Mécanique des Fluides et d'Acoustique (URA 263), \\ 69131 Ecully Cedex, France \\ (2) S.N.E.C.M.A., Centre de Villaroche, 77550 Moissy Cramayel, France
}

(Received 11 April 1991, revised 24 September 1991, accepted 9 January 1992)

\begin{abstract}
Résumé. - Les niveaux élevés de température atteints à la sortie des chambres de combustion des moteurs d'avions modernes, et les limitations pratiques des matériaux nouveaux, imposent un refroidissement efficace des aubages de turbines. L'optimisation du refroidissement nécessite une prédiction correcte des pertes aérodynamiques et des transferts de chaleur sur les aubages de turbines. Un nouveau code de calcul de couche limite compressible aérothermique a été développé. La formulation comprend une procédure d'interaction forte entre les écoulements visqueux et non visqueux, qui accroît les propriétés de stabilité du code. Les équations de couche limite, associées à l'équation d'énergie, sont résolues selon le schéma implicite de Keller. Le couplage des écoulements visqueux-non visqueux est mis en œuvre en joignant une équation d'interaction de nature elliptique. Le système complet d'équations est résolu par une procédure de balayages amont-aval. Cette technique contribue à stabiliser la méthode et permet le calcul de régions avec de forts gradients de pression adverse, des poches de décollement et des injections dans le cas de refroidissement par film. Des comparaisons entre les résultats expérimentaux et théoriques sont présentées. Des écoulements avec transfert de chaleur sont calculés pour plusieurs cas tels que des plaques planes avec de forts gradients de pression et des couches limites sur des aubages de turbines. Un accord satisfaisant entre le calcul et l'expérience est observé, montrant la précision et la robustesse du code de calcul.
\end{abstract}

\footnotetext{
Abstract. - The high temperature level reached at the exit of combustion chambers of modern aircraft engines and the practical limitations of advanced materials, demand efficient cooling of turbine blades. Optimization of the cooling requires an accurate prediction of aerodynamic losses and heat transfer on turbine blades. A new two-dimensional compressible, aerothermal boundary layer code has been developed. The formulation includes strong viscous-inviscid interaction, which enhances the stability properties of the code. The boundary layer equations associated with the energy equation are solved with an implicit Keller-box scheme. Viscous-inviscid flow coupling is performed by adding an interaction which has an elliptic character. The complete system of equations is solved by a multi-pass procedure. This technique contributes to the stabilization of the method and allows the computation of regions with strong adverse pressure gradients, separation bubbles and injections in case of film cooling. Comparisons between experimental and theoretical results are provided. Flow characteristics including heat transfer were computed for several cases such as flat plates with strong pressure gradients, and turbine blade boundary layers. Good agreement between computation and experiment is observed, demonstrating the high accuracy and robustness of the code.
} 


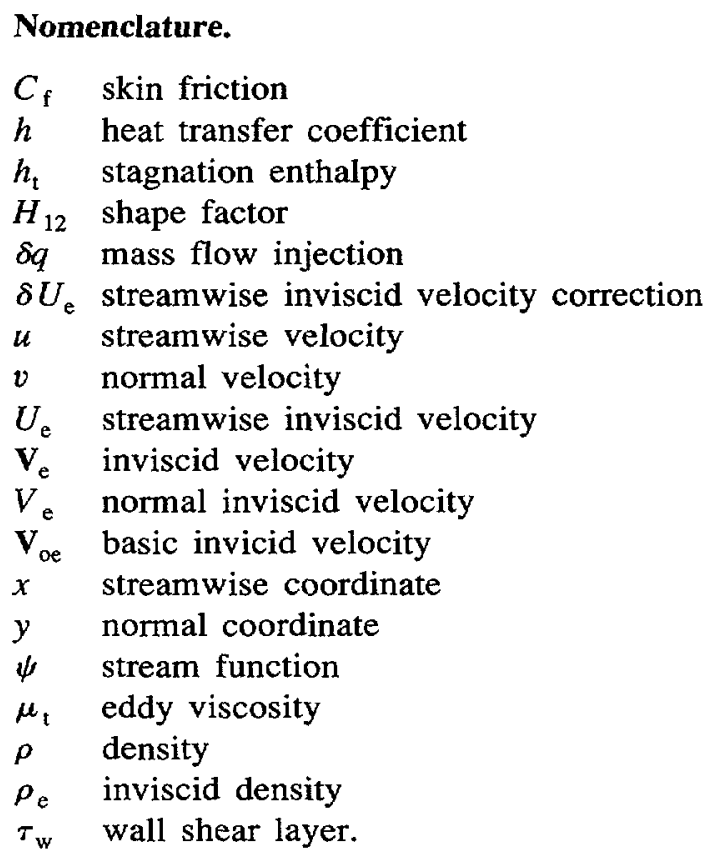

\section{Introduction.}

High temperature levels reached in modern high pressure turbines of aircraft engines require effective cooling. Film cooling has proved its ability to protect the blades and the endwalls from the hot upstream flow. However, the cooling jets induce important flow disturbances. In particular the losses and heat transfer associated with the viscous layers on the blades are modified. Determining the best compromise between thermal strains and aerodynamical performances is a major objective for turbine aerothermal design. S.N.E.C.M.A. has initiated an extensive experimental and theoretical research program on this subject.

A modelisation of the viscous flow on film cooled turbine blades is developed at Ecole Centrale de Lyon, Kulisa [1]. This approach is based on the combined use of an aerothermal wall layer computation and a three-dimensional jet code. The present paper explains the preliminary development of the wall layer model, without jets effects.

Although turbine blade flows are characterized by strong accelerations, adverse pressure gradients and separated regions may occur: leading edge separation bubble on the pressure side, shock-wave impingement, and, for film cooled blades, recirculation in the jet region. High stability properties of the numerical method are then required. For this purpose, the formulation, based on the two-dimensional boundary layer equations, includes a strong viscous-inviscid interaction model, which reinforces the stability.

The code was tested on various flow configurations. Results for laminar and turbulent layers including separation on flat plates and turbine blades are compared to measurements. Special attention is paid on the robustness and the versatility of the numerical method.

\section{Viscous flow model.}

The objective of this work is the modelisation of the two-dimensional compressible thermal wall layer flows on turbine blades. The viscous flow model is described hereafter; it is based on the turbulent Reynolds-averaged boundary layer equations coupled with an inviscid flow 
model. The thin-layer hypothesis will be discussed in paragraph 3 , in connection with viscousinviscid interaction procedure.

2.1 BASIC EQuATIONS. - The boundary layer equations system is written for a compressible flow. The conservation equation for the stagnation enthalpy is added to take into account thermal effects. In these equations, a change of variables substitutes the stream function $\psi$ to the normal velocity component $v$ :

$$
\rho v=-\frac{\partial \psi}{\partial x}
$$

Then the partial differential equations for the viscous flow can be written in the following form :

$$
\left\{\begin{array}{l}
\rho u=\frac{\partial \psi}{\partial y} \\
\frac{\partial \psi}{\partial y} \frac{\partial u}{\partial x}-\frac{\partial \psi}{\partial x} \frac{\partial u}{\partial y}-2 K_{x} \frac{\partial \psi}{\partial x} u=\rho_{\mathrm{e}} U_{\mathrm{e}} \frac{\mathrm{d} U_{\mathrm{e}}}{\mathrm{d} x}+\frac{\partial}{\partial y}\left[\mu \frac{\partial u}{\partial y}-\overline{\rho u^{\prime} v^{\prime}}\right] \\
\frac{\partial \psi}{\partial y} \frac{\partial h_{\mathrm{t}}}{\partial x}-\frac{\partial \psi}{\partial x} \frac{\partial h_{\mathrm{t}}}{\partial y}=\frac{\partial}{\partial y}\left[\mu u \frac{\partial u}{\partial y}-q_{y}-\overline{\rho v^{\prime} h_{\mathrm{t}}^{\prime}}\right] .
\end{array}\right.
$$

$K_{x} \frac{\partial \psi}{\partial x} u$ gives the wall curvature effect, where $K_{x}$ is the inverse of the wall curvature radius. $q_{y}$ is the heat flux, given as a function of the static temperature, by the Fourier's law.

The unknowns, directly related to the system (2), are $\left[\psi, u, h_{\mathrm{t}}\right]$. However, the inviscid velocity $U_{\mathrm{e}}$ is considered as an additional unknown. The closure of system (2) requires an additional equation; this equation relates the inviscid velocity $U_{\mathrm{e}}$ to the development of the viscous wall layer, and will be described in paragraph 3 .

The equations are written for a compressible flow, but the density $\rho$ is not considered as a global unknown, as $\left[\psi, u, h_{\mathrm{t}}\right]$. The nature of the fluid does not appear explicitly in the equations. This feature may be used in order to treat other fluids than perfect gases, without any modification of the equations. However, in this paper, the density will be calculated from the perfect gas law, as a function of the stagnation enthalpy and velocity.

Classical boundary conditions are applied to the system of equations (2) : no-slip condition and thermal conditions (either enthalpy or heat flux) are given at the wall; continuity with the inviscid flow at the outer edge of the boundary layer is ensured.

2.2 Turbulence Closure. - The complex phenomena which exist in viscous flows on turbine blades, require advanced turbulence models. But in this preliminary development of the method, the objective is to demonstrate its capabilities. Thus a simple turbulence model was introduced in the code.

The turbulence model is based on the Boussinesq hypothesis and thus uses the eddyviscosity concept $\mu_{\mathrm{t}}$ through the standard two-layers model of Cebeci-Smith [2].

A correction of the eddy-viscosity is applied to take into account the wall curvature effects $[3,4]$. The transition from laminar to turbulent flow is performed with the intermittency function, given by Dhawan and Narashima [5]. Usual experimental correlations for the onset and the end of transition are not suitable for turbine flows, thus they will be specified into the computation.

\section{Viscous-inviscid interaction.}

In most cases, direct calculation of turbine blade boundary layers cannot be realized in a satisfactory way, if the inviscid velocity at the outer edge of the boundary layer is prescribed. 
This is particulary true when strong interactions exit between the inviscid and the viscous flows ; either the static pressure or the inviscid velocity distribution has then to be corrected, Lebcuf, Kulisa, El Marjani [6]. Moreover, the parabolic nature of the boundary layer equations does not allow stable computation in separated flows.

To answer the purpose of stable computation, methods were developed to deal with the viscous-inviscid interactions, Veldman [7], Le Balleur [8, 9], Delery and Marvin [10], Edwards [11]. The coupling technique, introduced in the present work, is now described.

3.1 WEAK AND STRONG INTERACTIONS. - The intensity of the mutual influence between the viscous and the inviscid flows leads to define the weak and the strong interactions, Le Balleur [8].

Weak interaction is characterized by an inviscid flow which prescribes the static pressure to the viscous flow, Van Dyke [12]. An iterative procedure, between the viscous and the inviscid flows calculations, may be used without any special treatment. Besides, the corrections are small and a very small number of iterations is necessary.

In strong interaction case, the viscous flow strongly disturbs the inviscid flow. Strictly, the static pressure gradient, normal to the wall, cannot be ignored across the viscous flow. During the iterations between the viscous and the inviscid computations, relaxations on transferred informations, are often needed, Le Balleur [8]. Moreover, an unique calculation method cannot be used for both separated and attached viscous layer. Indeed, it is usual to perform a direct computation, with $U_{\mathrm{e}}$ prescribed, as long as the viscous layer is attached. Inverse computation, which prescribes a viscous information (e.g. : displacement thickness), is often used in separated regions. This technique has been widely developed by Le Balleur [8].

The present approach is based on the strong interaction concept. To avoid the difficulties related to the use of both direct and inverse modes in the same code, the viscous and the inviscid flows are considered simultaneously.

3.2 CHOICE OF THE COUPLING BOUNDARY. - We have developed an overlapping coupling method, in the sense that only one calculation domain is considered for the resolution of the viscous and the inviscid flows equations. In this case, the inviscid flow close to the wall is a fictitious flow which has no physical reality.

We have now to define the coupling boundary where the two flows exchange information. The choice of this boundary is very important and determines the behaviour of the viscous flow, in the coupling approach, Le Balleur [13]. More precisely, for a given supersonic inviscid Mach number, the coupling may introduce either an elliptical or a hyperbolical nature of the viscous flow equations depending on the location of the information transfer. Delery [10] defines the subcritical or supercritical character of the viscous layer if, in a global manner, it behaves respectively as a subsonic or as a supersonic flow.

If the coupling is realized at the boundary $y=\delta^{*}$ (the displacement thickness) or at $y=\delta$ (the outer edge of the boundary layer) this supercritical behaviour appears for the inviscid Mach number greater than 2 or 1.4. On the contrary, an information transfer at the wall ensures a subcritical behaviour of the viscous layer, whatever the inviscid Mach number may be.

We have chosen to locate the coupling boundary at the wall. This maintains a subcritical behaviour of the coupled problem, even if the inviscid Mach number is supersonic. As a consequence, the calculation method must take into account this underlying elliptic aspect, which is related to the subcritical character of the coupled problem.

3.3 STRONG VISCOUS-INVISCID INTERACTION MODEL. - In this paper, the viscous-inviscid interaction is based on the use of the real flow mass defect with regard to the inviscid flow 
which is defined up to the wall, Van Dyke [12]. To simulate this viscous layer influence on the inviscid flow, the inviscid mass flow must be corrected by the integral mass flow defect $\delta q$ :

$$
\delta q=\int_{0}^{\delta}\left(\rho_{\mathrm{e}} U_{\mathrm{e}}-\rho u\right) \mathrm{d} y .
$$

These $\delta q$ injections are introduced at the wall in the inviscid flow computation. The mass flow distribution induces, in the inviscid flow, a normal to the wall velocity component $V$ e. A defect form of the continuity equation is integrated from the wall to the outer edge of the viscous layer. This equation relates $V_{\mathrm{e}}$ to the mass flow injections $\delta q$ :

$$
\left(\rho_{\mathrm{e}} V_{\mathrm{e}}\right)_{y=0}=\frac{\partial}{\partial x}(\delta q) \text {. }
$$

This interaction model mainly modifies the location and the curvature of the inviscid streamlines.

The advantage of this formulation is that the use of these mass flow injections in the coupling technique is suitable for the future film cooling real injections, while the standard wall displacement technique would hardly accomodate this feature.

Moreover, a perturbation of the static pressure gradient, normal to the wall, is induced. Strictly, a gradient $\frac{\partial P}{\partial y}$ exists. In the present work, in order to retain the thin layer hypothesis, we shall consider the static pressure as constant across the viscous layer and equal to its particular wall value induced by the coupling.

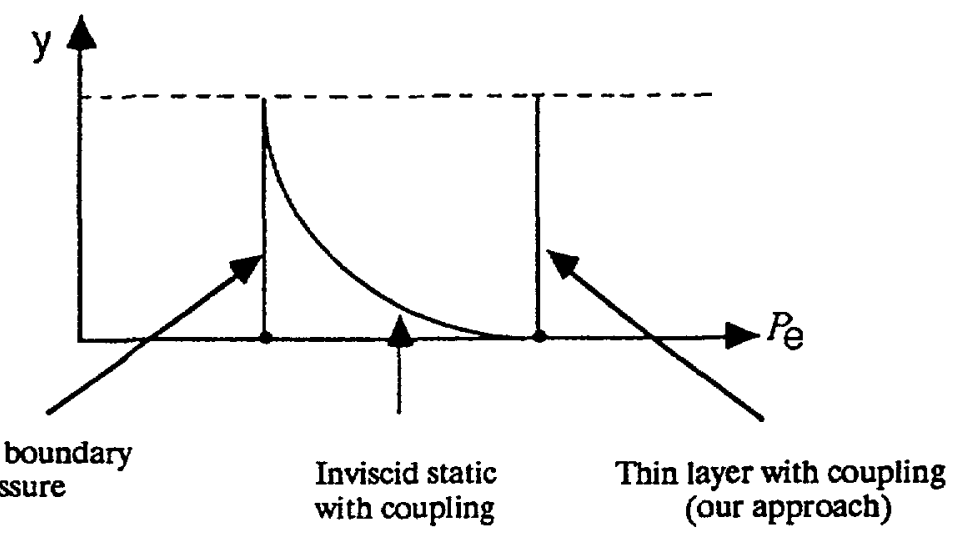

Indeed, the basic idea is that the viscous flow is mainly determined by the static pressure at the wall rather than at the outer edge of the viscous layer. In practice, the inviscid wall static pressure correction is taken into account, in the viscous flow computation, by a correction of the inviscid velocity $\delta U_{\mathrm{e}}$.

In brief, the boundary layer equations (2) have been explained as well as the relation between the viscous and the inviscid flow (3) and (4). We have now to present the inviscid flow equations. The form of the interaction model, requires the determination of the inviscid flow at the wall only. Thus, a simplified modelisation of the inviscid flow was performed and is presented in the next part.

3.4 SIMPLIFIED INVISCID FLOW MODEL. - This model uses an integral formulation for the correction of the inviscid flow. Denoting by $V_{o e}$ the basic inviscid flow velocity, and 
$\delta V_{e}$ its change induced by viscous effects, we consider the new inviscid velocity $\mathrm{V}_{\mathrm{e}}$ :

$$
\mathbf{V}_{\mathrm{e}}=\mathbf{V}_{\mathrm{oe}}+\delta \mathbf{V}_{\mathrm{e}}
$$

The Poincaré's formula, Jacob [14], may be used to compute the velocity induced at a point $\mathrm{M}$ of the domain limited by a boundary $\sigma$, by normal injections on $\sigma$ :

$$
\begin{aligned}
E V(M)=\operatorname{rot}\left[\iint_{\sigma} \frac{y_{Q} \wedge \mathrm{V}(\mathrm{Q})}{\overline{\mathrm{MQ}}} \mathrm{d} \sigma+\right. & \iiint_{0} \frac{\operatorname{rot} \mathrm{V}(\mathrm{Q})}{\overline{\mathrm{MQ}} \mathrm{d} v]-} \\
& -\operatorname{grad}\left[\iint_{\sigma} \frac{\mathrm{V}(\mathrm{Q}) \cdot \mathbf{y}_{\mathrm{Q}}}{\overline{\mathrm{MQ}}} \mathrm{d} \sigma+\iiint_{v} \frac{\operatorname{div} \mathrm{V}(\mathrm{Q})}{\overline{\mathrm{MQ}}} \mathrm{d} v\right] .
\end{aligned}
$$

In the frame of an incompressible and irrotational hypothesis for $\delta V_{e}$, [6], an approximated form of (6) may be obtained :

$$
\delta \mathrm{V}_{\mathrm{e}}(\mathrm{M})=-\frac{1}{2 \pi} \operatorname{grad}_{\mathrm{M}} \iint_{\sigma} \frac{V_{\mathrm{e}}(\mathrm{Q})}{\mathrm{MQ}} \mathrm{d} \sigma .
$$

The nature of the flow and the small perturbations concept in connection with a basic flow, are not present in this equation which is elliptic. The correction of the velocity, at the wall, may be calculated whatever the nature of the basic flow is. The idea of corrections or perturbations is introduced in equation (5). It is sufficient that these corrections are small to be considered as incompressible and irrotational.

If we consider a semi-infinite two-dimensional flow on a flat wall, the change of the streamwise velocity component $\delta U_{\mathrm{e}}$ at a point $\mathrm{M}$ located at the wall may be obtained from (7) :

$$
\delta U_{\mathrm{e}}=-\frac{1}{\pi} \int_{L} \frac{V_{\mathrm{e}}(x)}{x-x_{\mathrm{M}}} \mathrm{d} x .
$$

$L$ is the contour of the wall where the viscous flow develops. A similar equation was used for incompressible boundary layers by Veldman [7] and Edwards [11]. This technique was introduced for secondary flows computations applied to multistage compressors, Lebœuf and Brochet [15].

Finally, substituting the velocity $V_{\mathrm{e}}$, given by equation (4), into equation (8) we obtain :

$$
\delta U_{\mathrm{e}}=-\frac{1}{\pi} \int_{L} \frac{1}{\rho_{\mathrm{e}}} \frac{\frac{\mathrm{d}}{\mathrm{d} x}(\delta q)}{x-x_{\mathrm{M}}} \mathrm{d} x .
$$

(9) is labelled interaction system, and relates the velocity corrections $\delta U_{\mathrm{e}}$ at each calculation point of the wall, to the mass flow defect distribution. For strong coupling applications, the corrections $\delta U_{\mathrm{e}}$ may be important and the assumption of incompressibility may not be justified. In practice, we do not use directly the equation (9), but the results of some previous iteration between two space-marching iterations. In fact, a correction $\mathrm{d}\left(\delta U_{\mathrm{e}}\right)$ is calculated and equation $(10)$ is obtained :

$$
\mathrm{d}\left(\delta U_{\mathrm{e}}\right)=-\frac{1}{\pi} \int_{L} \frac{1}{\rho_{\mathrm{e}}} \frac{\mathrm{d}\left(\frac{\mathrm{d}}{\mathrm{d} x}(\delta q)\right)}{x-x_{\mathrm{M}}} \mathrm{d} x .
$$


At convergence, the corrections $\mathrm{d}\left(\delta U_{\mathrm{e}}\right)$ approach zero, which is compatible with the assumption of an incompressible correction.

The interaction system (10), combined with the definition of $\delta q,(3)$, allows us to close the boundary equations (2). The elliptic character of the interaction system (10) appears for all the points $M$ at the wall, as the integral domain extends from upstream to downstream of $M$.

\section{Numerical method.}

The objective of the method is a detailed and local analysis of the flow. Thus a differential method was chosen for the numerical solution. The equations presented in paragraphs 2 and 3 , are discretized according to Keller's box method [16], which is a second order implicit scheme. The main feature of this scheme is to reduce the derivatives order in the equations. As a consequence, only first order derivatives still exist. Keller's box scheme, applied to system (2), leads to introduction of two additional variables $\tau$ and $Q$, thus eliminating the second order derivatives of $u$ and $h_{\mathrm{t}}$ :

$$
\left\{\begin{array}{l}
\tau=\mu \frac{\partial u}{\partial y}-\rho \overline{u^{\prime} v^{\prime}} \\
Q=\mu u \frac{\partial u}{\partial y}-q_{y}-\overline{\rho v^{\prime} h_{t}^{\prime}} .
\end{array}\right.
$$

The following system is then obtained :

$$
\left\{\begin{array}{l}
\rho u=\frac{\partial \psi}{\partial y} \\
\frac{\partial \psi}{\partial y} \frac{\partial u}{\partial x}-\frac{\partial \psi}{\partial x} \frac{\partial u}{\partial y}-2 K_{x} \frac{\partial \psi}{\partial x} u=\rho_{\mathrm{e}} U_{\mathrm{e}} \frac{\mathrm{d} U_{\mathrm{e}}}{\mathrm{d} x}+\frac{\partial \tau}{\partial y} \\
\frac{\partial \psi}{\partial y} \frac{\partial h_{t}}{\partial x}-\frac{\partial \psi}{\partial x} \frac{\partial h_{t}}{\partial y}=\frac{\partial Q}{\partial y} \\
\tau=\mu \frac{\partial u}{\partial y}-\overline{\rho u^{\prime} v^{\prime}} \\
Q=\mu u \frac{\partial u}{\partial y}-q_{y}-\overline{\rho v^{\prime} h_{\mathrm{t}}^{\prime}} .
\end{array}\right.
$$

The unknowns are now: $\left[\psi, u, h_{\mathrm{t}}, \tau, Q\right]$, plus the inviscid velocity $U_{\mathrm{e}}$, related to the viscous-inviscid interaction.

The new system is more complicated from an algebraic point of view, because Keller's box scheme yields more equations and more unknowns. Nevertheless, only first order derivatives exist, the discretization on a box introduces only the variables at the four grid points of this box, leading to a very compact scheme.

The versatility of the method may be remarked for the implementation of another turbulence model, even if it is not based on the Boussinesq hypothesis. This feature is obtained because the turbulent correlations are isolated in the last two equations.

In practice, the system (11) is discretized on a box, defined by the streamwise locations (I-1) and (I); the variables are known in the upstream station (I-1) and unknown in the downstream station (I). The computation uses a space-marching method.

Note that the inviscid velocity $U_{\mathrm{e}}$ at the present station I only is needed. It is then useless to solve the complete interaction system (10) at the current station I. As a matter of fact, only the $I$-th line of this system needs to be joined to the system (11). These resulting 6 equations 
are then solved simultaneously, according to the strong viscous-inviscid interaction model, with the space-marching method.

Let us now precise this coupling equation. The interaction system (10) is discretized as follow :

$$
\left[\mathrm{d}\left(\delta U_{\mathrm{e}_{j}}\right)\right]=[\mathcal{A}(J, K)]\left[\mathrm{d}\left(\delta q_{K}\right)\right] .
$$

This $I$-th line is then written as :

$$
\begin{aligned}
\mathrm{d}\left(\delta U_{\mathrm{e}}\right)^{n+1}= & \sum_{K=1, I-1} \mathcal{A}(I, K) \mathrm{d}\left(\delta q_{K}\right)^{n+1}+\mathcal{A}(I, I) \mathrm{d}\left(\delta q_{I}\right)^{n+1}+ \\
& +\sum_{K=I+1, N} \mathcal{A}(I, K) \mathrm{d}\left(\delta q_{K}\right)^{n}
\end{aligned}
$$

with

$$
\left\{\begin{array}{l}
U_{\mathrm{e}}=U_{\mathrm{oe}}+\delta U_{\mathrm{e}} \\
\delta q=\int_{0}^{\delta}\left(\rho_{\mathrm{e}} U_{\mathrm{e}}-\rho u\right) \mathrm{d} y
\end{array}\right.
$$

The elliptic effect, introduced by this coupling equation, is emphasized by the known upstream mass flows and the unknown downstream mass flows influence. In order to update the downstream injections, the space-marching method will be iteratively repeated. This iterative procedure is equivalent to solving the interaction system with a Gauss-Seidel method. It is well known that a Gauss-Seidel method is very efficient for an iterative treatment of $M$-matrices, as the matrix $\mathcal{A}$ in (12). It allows a simultaneous treatment with the viscous layer equations system (11). For a given upstream-downstream sweep number $n$, the final system, that we have to solve, is written as :

$$
\left\{\begin{array}{l}
\text { System (10) } \\
\mathrm{d}\left(\delta U_{\mathrm{e}_{l}}\right)^{n}=\sum_{K=1,1-1} \mathcal{A}(I, K) \mathrm{d}\left(\delta q_{K}\right)^{n}+\mathcal{A}(I, I) \mathrm{d}\left(\delta q_{I}\right)^{n}+\sum_{K=I+1, N} \mathcal{A}(I, K) \mathrm{d}\left(\delta q_{K}\right)^{n-1}
\end{array}\right.
$$

The solution technique of this system must now be specified, at a given station I during a sweep. The equations are non-linear, and the iterative Newton-Raphson method is used.

\section{Computational results.}

The code was tested on various flows using direct mode, when possible, and coupled mode [1]. In this part, we pay a particular attention to the description and the analysis of the results obtained for strong viscous-inviscid interaction flows. Thereby we first present two cases of flat plate flows under strong adverse pressure gradient, which point out the interest of the viscous-inviscid interaction. Then, the code is evaluated on two cases of turbine blade flows. These tests exhibit the major difficulties which can be encountered in reality : laminarturbulent transition, heat transfer, strong deceleration due to the impingement of a shock wave, separation bubble near the leading edge of the pressure side.

5.1 LAMINAR SEPARATION BUBBLE. - The first case is provided by numerical results obtained by Briley [17]. Calculation using two-dimensional incompressible Navier-Stokes equations was performed for a laminar flow. Briley simulates a deceleration leading to a 
separation, followed by a region where the external velocity is constant in order to induce a reattachment. The laminar nature of the flow emphasizes the advantage of the coupling method.

Distributions of the freestream velocity outside the viscous layer, given by Briley, and of the inviscid velocity recalculated at the wall by the interaction equation are shown in figure 1 . As the separation point is reached, the difference between the two velocities becomes larger. Indeed the increase of the boundary layer displacement thickness induces strong streamline curvature and therefore a noticeable static pressure variation (expressed herein in terms of inviscid velocity), in the direction normal to the wall.

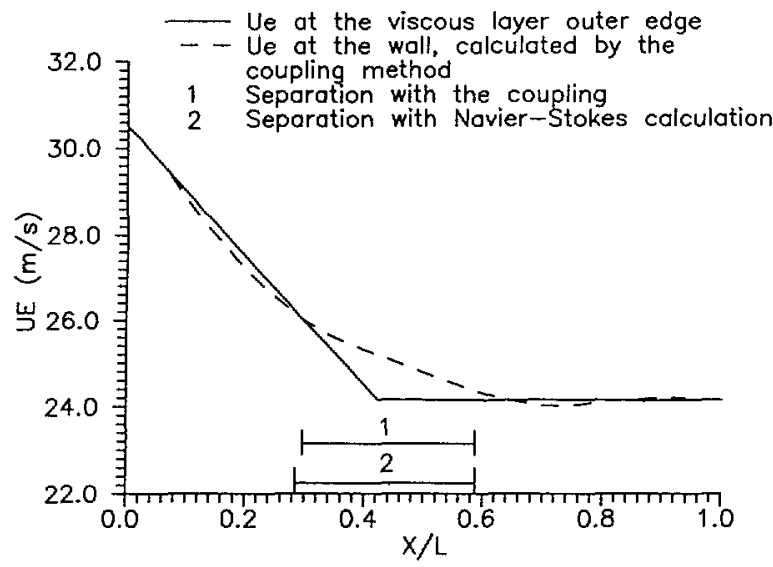

Fig. 1. - Variation of inviscid velocity $U_{\mathrm{e}}$ versus non-dimensional streamwise distance.

The shape factor $H_{12}$ and the wall shear stress $\tau_{\mathrm{w}}$ variations are shown in figures 2 and 3 . The results obtained with the present method and with the Navier-Stokes equations are very close. In particular, the locations of the separation and reattachment points are quite similar. Thus it seems that the viscous-inviscid interaction method provides most of the streamwise

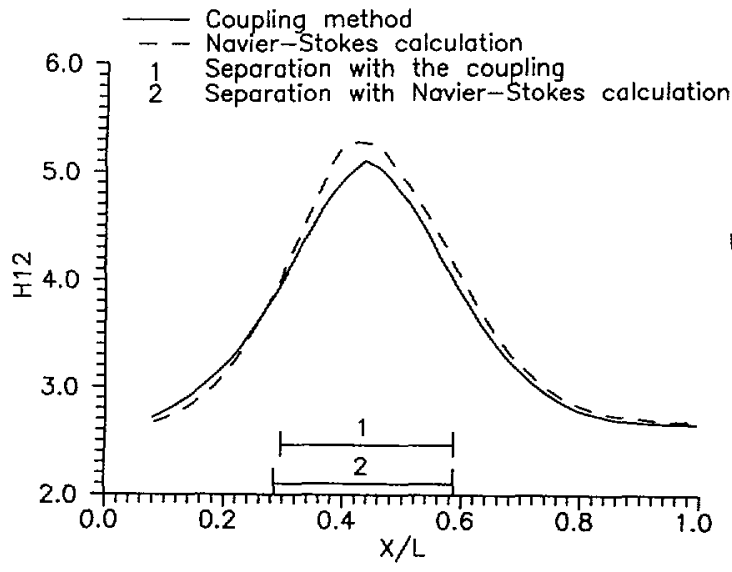

Fig. 2.

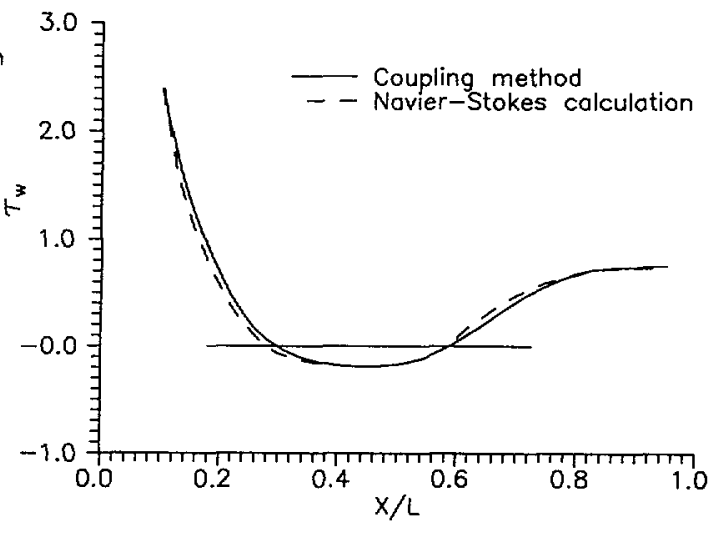

Fig. 3 .

Fig. 2. - Variation of the shape factor $H_{12}$ versus non-dimensional streamwise distance.

Fig. 3. - Variation of the wall shear stress versus non-dimensional streamwise distance. 
elliptic effects which are captured in the Navier-Stokes equations. In this case, the coupling method gives results comparable to those obtained with Navier-Stokes calculation while retaining a more simple modelisation level and much shorter calculation times. The stability reinforced by the viscous-inviscid interaction has been verified.

5.2 TuRBUlent SEPARATED Flow. - An incompressible turbulent viscous layer developing on a flat plate was studied in a wind-tunnel by Simpson et al. [18]. The flow is first accelerated and then sufficiently decelerated to produce a strong separation. Reverse flow velocities of the order of $20 \%$ of the external velocity are observed.

The experimental inviscid velocity distribution measured at the outer edge of the layer is compared to the inviscid velocity distribution at the wall, calculated by the interaction model, figure 4. Again, differences may be observed along the deceleration which become more and more important as the separation zone approaches. This phenomenon reveals, as in the previous case, the existence of the static pressure gradient (or herein inviscid velocity gradient) normal to the wall which results from the viscous-inviscid flow interaction.

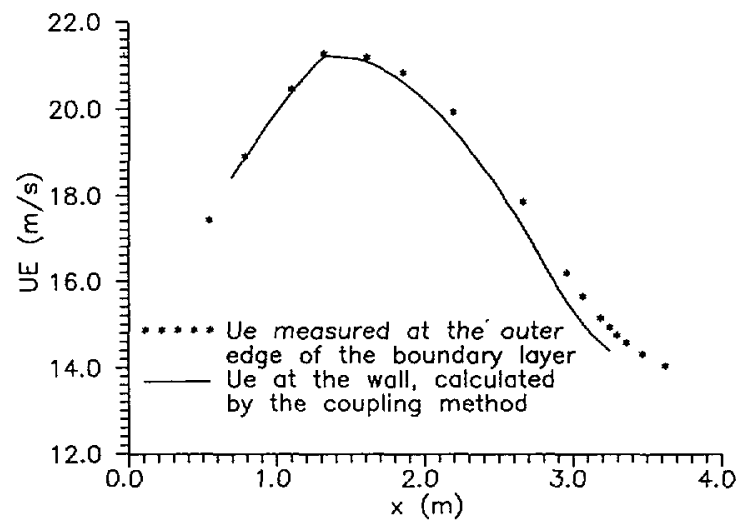

Fig. 4. - Variation of inviscid velocity $U_{\mathrm{e}}$.

As the last calculation station is located in the separation area, a downstream boundary condition is introduced explicitly as in any elliptic problem, by imposing the mass flow $\delta q$.

The results in terms of shape factor $H_{12}$ and skin friction coefficient (Fig. 5 and Fig. 6) are close to the experimental data and enhance the predominant effects of the streamwise inviscid velocity gradients at the wall. It is surprising to obtain such good results with only a mixing length turbulence model. In fact the flow behaviour is mainly determined by the increase of the boundary layer displacement thickness. The coupling method enables to take into account this predominant effect. However, a more detailed comparison, based on velocity profiles for example, is not as good and better accuracy requires a turbulence closure suitable to separated flows.

It should be noted that the direct mode calculation cannot restore the shape factor increase during the deceleration. Besides, after the separation point, the calculation diverges.

The stability and the efficiency of the coupling method have been demonstrated for a large turbulent separated case, whereas a standard boundary layer approach, using the direct mode, is not suitable.

5.3 TURBINE BLADE FLOWS. - We now consider the behaviour of the method on real cases of turbine blade, with strong interactions such as : strong deceleration due to the effect of shock-wave impingement, pressure side separation bubble. 


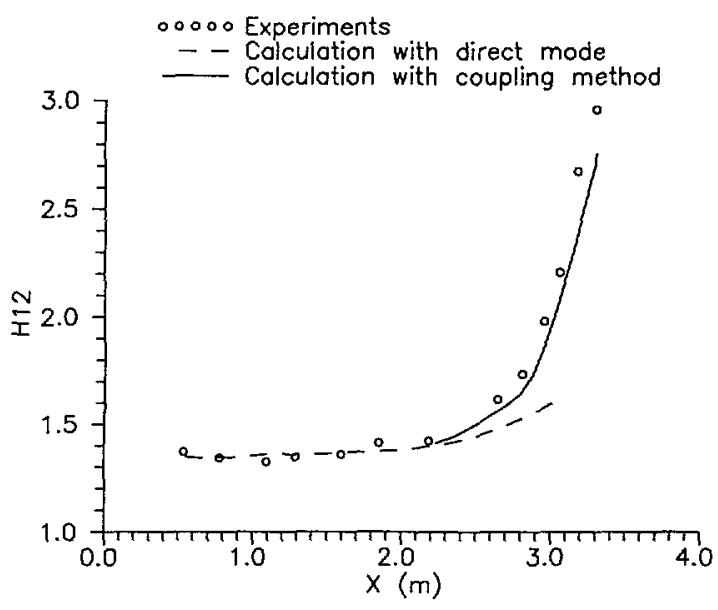

Fig. 5.

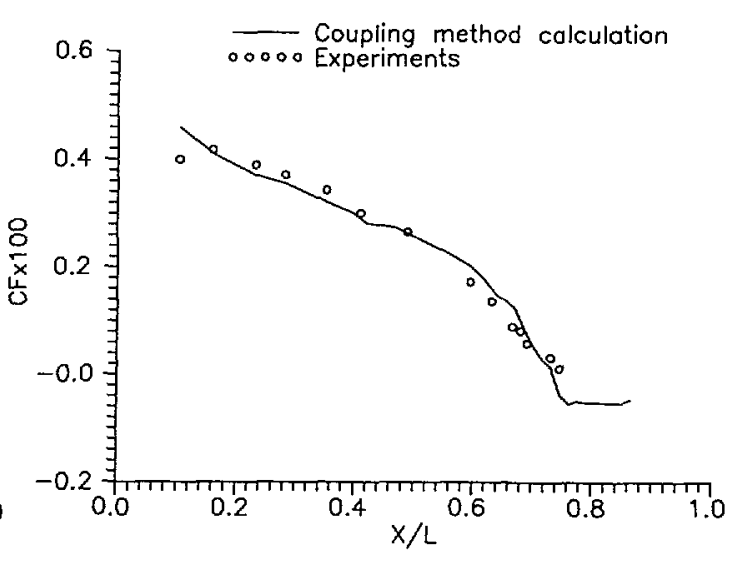

Fig. 6 .

Fig. 5. - Variation of the shape factor $H_{12}$.

Fig. 6. - Calculated skin friction coefficient versus non-dimensional streamwise distance.

In most cases, the wall layers cannot be evaluated in direct mode. However, the use of coupling method is not straightforward in this context. The basic inviscid velocity distributions $V_{o e}$ are deduced from pressure measurements at the wall and thus already take into account the unknown viscous layer effects. The role of the coupling equation (13) is here to locally readjust the streamwise pressure gradient at the wall. This improves mainly the stability of the method. This method is valid for small pressure corrections.

The experimental results considered here are provided from a transonic linear cascade at the Von Karman Institute, Arts [19, 20]. Both static pressure on the blade and heat transfer coefficients were measured.

5.3.1 Turbine rotor blade flow. - The flow considered develops on the profile of a high pressure turbine rotor [19]. The Mach number distribution is plotted in figure 7 for the suction and the pressure sides. The wall temperature reaches $296.8 \mathrm{~K}$, whereas the external flow temperature is $422.6 \mathrm{~K}$.

5.3.1.1 Suction side. - The flow is transonic. The calculation was performed by locating the transition zone between $70 \%$ and $80 \%$ of the profile length.

The heat transfer coefficient evolution $h$, presented in figure 8 , is in agreement with the experimental one. In particular, the increase of $h$ during the laminar-turbulent transition is well predicted. As strong trailing edge deceleration is observed experimentally. The coupling equation allows to calculate properly the flow development, by a smooth readjustment of the static pressure gradient at the wall.

5.3.1.2 Pressure side. - On the pressure side, the laminar-turbulent transition is located between $11 \%$ and $13 \%$ of the profile length.

One of the difficulties of this flow occurs in the vicinity of the leading edge. The laminar flow deceleration results in a separation region and induces a sudden decrease of the heat transfer coefficient $h$, figure 9. The transition is located in this separation zone ; it leads to the viscous layer reattachment and thus to the strong increase of $h$. Although the complete rise of the coefficient $h$ up to the experimental point is not reflected by the calculation, the physical phenomena are quite well reproduced. 


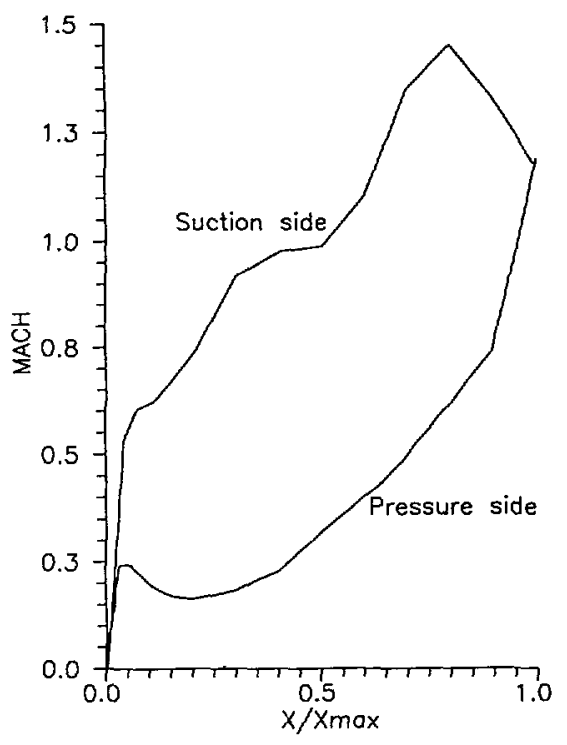

Fig. 7. - Variation of Mach number for the turbine rotor blade flow.

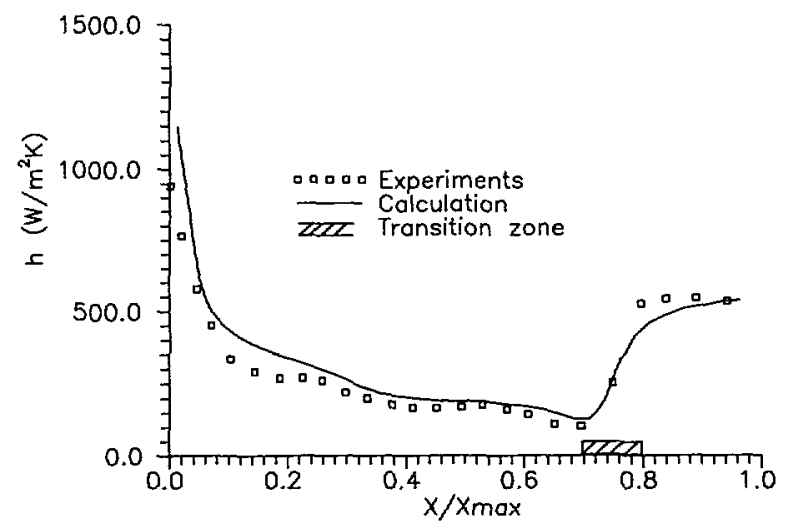

Fig. 8. - Variation of heat transfer coefficient on the suction side.

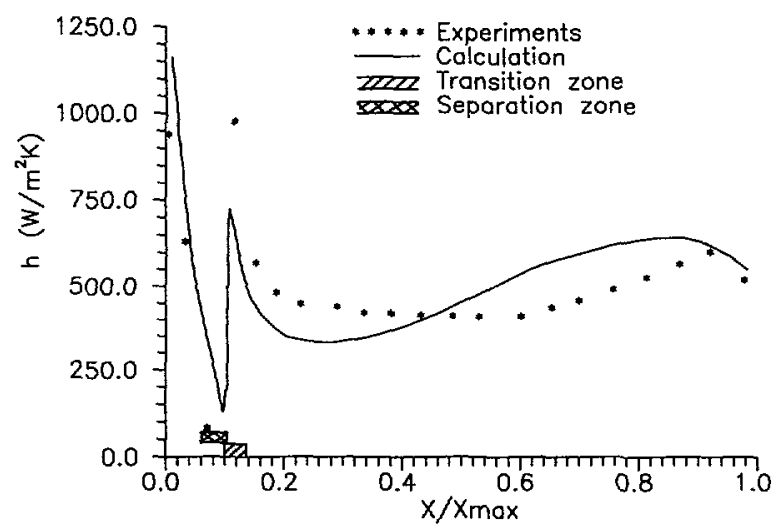

Fig. 9. - Variation of heat transfer coefficient on the pressure side. 
Far downstream, the calculation presents some discrepancies with the experiment. The strong acceleration may be the cause of this behaviour. A relaminarization phenomenon, induced by this acceleration, may maintain a quasi-constant level of $h$. The turbulence model, used here, is not able, of course, to take into account of this phenomenon.

Skin friction measurements are not available; nevertheless, the evolution of the calculated skin friction is presented figure 10 in order to locate the separation zone.

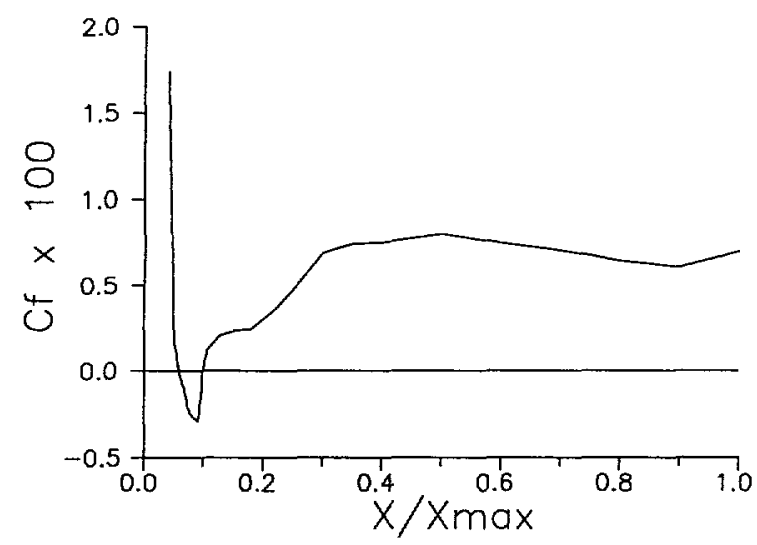

Fig. 10. - Variation of calculated skin friction on the pressure side.

5.3.2 Turbine stator blade flow. - The flow develops on a high pressure turbine stator blade [20]. The Mach number distribution is shown figure 11, on the suction and pressure sides. The wall temperature reaches $296.8 \mathrm{~K}$, whereas the external flow temperature is $422.6 \mathrm{~K}$.

5.3.2.1 Suction side. - On the suction side, a strong deceleration is due to the impingement of a weak shock. Near the trailing edge, a second deceleration occurs. The laminar-turbulent transition region is located at the first velocity peak, between $50 \%$ and $56 \%$ of the profile length.

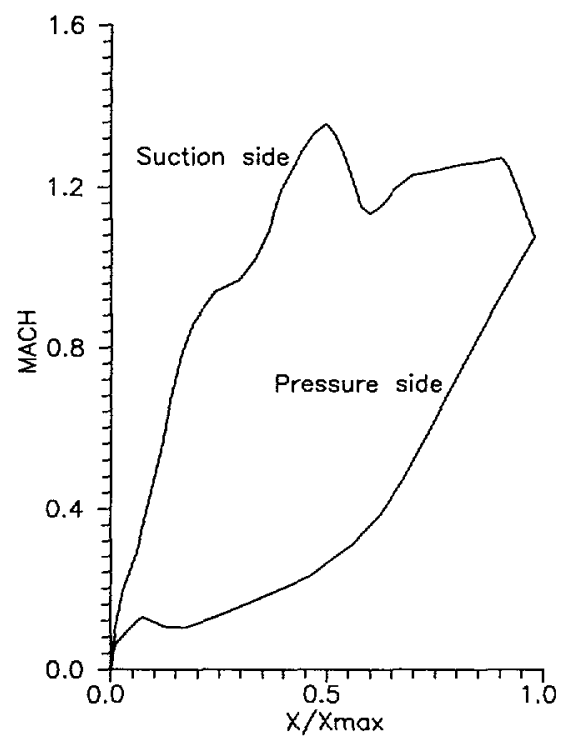

Fig. 11. - Variation of Mach number for the turbine stator blade flow. 
The variation of the heat transfer coefficient $h$ is given figure 12. The decrease of $h$, induced by the acceleration effect on the laminar viscous layer is well predicted. Nevertheless, the calculation is not able to produced the plateau of $h$. Further downstream, the increase of $h$, during the transition zone and the two decelerations, is well reproduced, despite the simplicity of the turbulence closure model.

5.3.2.2 Pressure side. - The transition zone is set at the velocity peak between $7 \%$ and $8 \%$ of the profile length.

For that case the leading edge deceleration is not sufficient to lead to the separation. The evolution of the heat transfer coefficient is shown in figure 13. Though the overall results are good, the strong acceleration is a borderline case for the turbulence model and can explain the high level of the calculated heat transfer coefficient. Indeed a relaminarization phenomenon (complete or partial) probably occurs which leads to a decrease of the heat transfer.

The capability of the method to treat real configurations on turbine blades has been shown. The physical evolutions are well reproduced and the stability of the numerical method has been demonstrated.

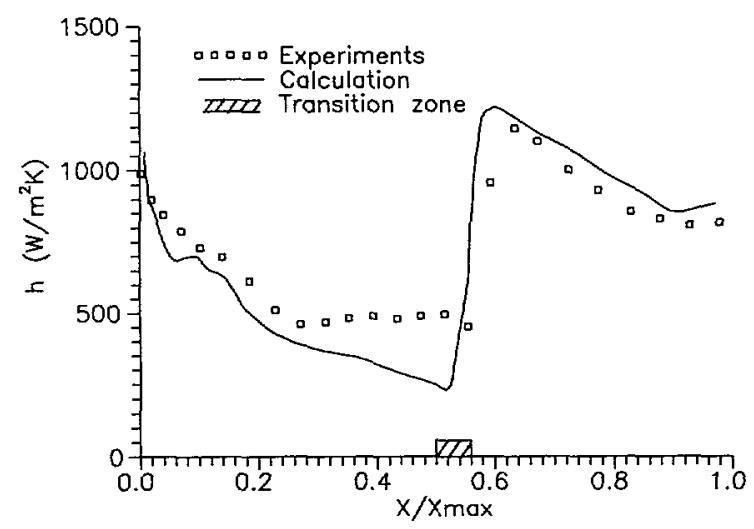

Fig. 12. - Variation of heat transfer coefficient $h$ on the suction side.

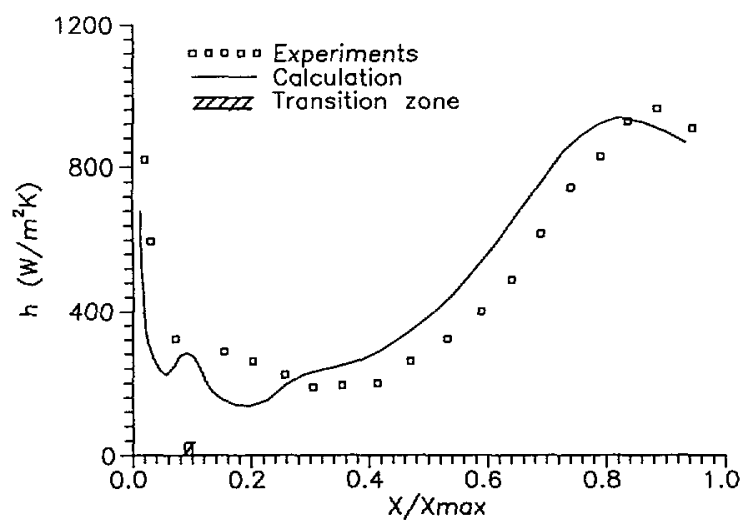

Fig. 13. - Variation of heat transfer coefficient $h$ on the pressure side. 


\section{Conclusions.}

A modelisation of viscous flows on turbine blades has been developed. It is based on boundary layer equations and a strong viscous-inviscid interaction model. Particular attention has been paid on numerical method in order to ensure the stability of the code, reinforced by the viscous-inviscid coupling.

The parabolic character of the boundary layer equations allows us to solve them with a space marching method. The elliptic effect, induced by the viscous-inviscid interaction and introduced by the coupling equation, requires the use of a multi-pass procedure which obviously increases the computation time. However, this coupling equation is necessary to ensure the stability of the method in the case of complex flows. In practice, no relaxation is necessary during this iterative process. Besides the increase in the computation time is small, the convergence of the sweeps is rapidly obtained ( 5 to 8 sweeps for complex flow case).

Tests were performed on complex flows. In particular, the capability of the method to treat real turbines blades configurations has been presented. The physical evolutions are respected and the stability of the numerical method has been demonstrated, even for leading edge separation bubble and strong decelerations due to shock wave impingement.

Future work will focus on the turbulence closure model which may be deficient when a strong acceleration or a separation occurs. The implementation of a more sophisticated model, facilited by the versatility of the Keller's box method, should improve the results. The second point concerns the interaction of the viscous layer with the film cooling jets.

The high stability of the method is encouraging for these purposes.

\section{Acknowledgements.}

We would like to thank S.N.E.C.M.A. and D.R.E.T. for the financial support of this study.

\section{References}

[1] Kulisa P., Etude théorique des écoulements visqueux en présence d'injections pariétales en vue du refroidissement des turbines, Thèse de Doctorat, Ecole Centrale de Lyon (1989).

[2] Cebeci T., Smith A. M. O., Analysis of turbulent boundary layer (Academic Press, 1974).

[3] BRADSHAw P., Effects of streamline curvature on turbulent flow, AGARDograph n 169 (1973).

[4] JohNSTON J. P., EIDE S. A., Turbulent boundary layers on centrifugal compressor blades: prediction of the effects of surface curvature and rotation, ASME, J. Fluids Eng. (1976).

[5] Dhawan S., Narasimha R., Some properties of boundary layer flow during the transition from laminar to turbulent motion, J. Fluid Mech. 3 (1958).

[6] Lebeuf F., Kulisa P., El Marjani A., Viscous computations in turbomachine on the use of integral equations, 5th International Conference on Numerical Methods in Laminar and Turbulent Flow, Montréal (1987).

[7] VeldMAN A. E. P., New, quasi-simultaneous method to calculate interacting boundary layers, AIAA J. 19 n 1 (1981).

[8] LE BALLEUR J. C., Calculs couplés visqueux-non visqueux incluant décollements et ondes de choc en écoulement bidimensionnel, AGARDograph LS-94.

[9] Le Balleur J. C., Calcul des écoulements à forte interaction au moyen de méthodes de couplage, AGARDograph CP-291, Computation of viscous-inviscid interaction (1980).

[10] Delery J., Marvin J. G., Shock-wave boundary layer interactions, AGARDograph AG-280 (1986). 
[11] EDWARDS D. E., Analysis of three-dimensional separated flow using interacting boundary layer theory, IUTAM Symposium « Boundary layer separation », F. T. Smith, S. N. Brown Eds. (Springer-Verlag, London, 1986).

[12] VAN DYKe M., Perturbation methods in fluids mechanics (Parabolic Press, 1975).

[13] Le Balleur J. C., Couplage visqueux-non visqueux : analyse du problème incluant décollements et ondes de choc, Rech. Aérosp. n 1977-6 (1977).

[14] JACOB C., Introduction mathématique à la mécanique des fluides (Gauthier-Villars, Paris, 1959).

[15] LEBEUF F., BROCHET J., The use of secondary flow computation in the compressor design process, Three-dimensional flow phenomena in fluid machinery, 32 (Hamed, Herring, Povinelli, ASME-FED, 1985).

[16] Keller H. B., A new difference scheme for parabolic problems, J. Bramble Ed. (Academic, NewYork, 1970).

[17] BRILEY W. R., A numerical study of laminar separation bubble using Navier-Stokes equations, $J$. Fluid Mech. 47 Part 4 (1971).

[18] Simpson R. L., Chew Y. T., ShivapRASAD B. Y., The structure of separating turbulent boundary layer. Part 1: Mean flow and Reynolds stress, J. Fluid Mech. 11 Part 3 (1981).

[19] ARTs T., Mesures des efficacités de film et des coefficients d'échange sur une aube rotorique $H . P$., Rapport I.V.K. CR 1985-01 (1985).

[20] ARTs T., Mesures des efficacités de film et des coefficients d'échange sur une aube rotorique H. P., Rapport I.V.K. CR 1986-05 (1986). 